\title{
ON WORDS AND WORLDS: COMMENTS ON THE ISARD AND SMITH PAPERS
}

\author{
GUNNAR OLSSON \\ University of Michigan
}

The following remarks are my comments on the exciting papers by Walter Isard and Tony Smith. ${ }^{1}$ I think their papers make a very nice piece, for in my mind they carry the seeds of their own destruction. From reading and discussing them we are therefore bound to learn, for to learn is to become so self-conscious about our work that we eventually negate it. Here-as so often recently-I am reminded of Hegel, who argued that the essential nature is to be the reverse of what it wants to be and who claimed that our activities pass into the opposite of what they immediately are.

Where in the Isard-Smith works do I see these seeds of self-destruction? I see them in several places, but nowhere as clearly as in their definitions of homomorphism, numerical representation, and separability of order structures. These definitions of homomorphism, counting, and identity are then reflected in all subsequent derivations and most clearly in the three axioms, which together constitute the theory of spatial discounting. It is in fact the clarity of the authors' presentations that makes us realize that whatever they say in their paper tells us more about the language and culture they are talking in than about the subject matter they are talking about. This is, of course, nothing peculiar, for it is generally the case that our thoughts and actions reflect the categorical scheme by which we impose mental structures on the phenomena with which we are dealing.

It is in this light that I shall evaluate Isard's claim that after a quarter of a century he finally has found a reasonable rationale for explaining gravity model trip behavior. This rationale is that of mathematical economics or-to use his own words-it is a maximizing rationale for ordinary people. This, then, is the structure of the net in which the authors try to catch-not themselves, for everybody knows that that is impossible-but all us others, that is, you and me, our children and our parents, those we think we love and those we think we hate. There are many problems built into this reasoning net, but the most forbidding are those connected with the principles of transitivity and separability.

These two principles of orderly reasoning go back a long time. Indeed they have never been specified more succinctly than in Leibniz's definition of identity and identity substitution. These principles are themselves embedded in the law of the excluded middle and they say that everything is identical to itself and nothing is identical to anything else. The problem here is how we can know that what you hold to be

${ }^{1}$ This written version follows rather closely the notes for my oral presentation at the 1974 RSA meeting in Chicago. 
separable and substitutable is what $I$ hold to be separable and substitutable. Rephrased, the question is how I can know that what I take to be the same is what you take to be the same as well.

In conventional formal reasoning of the type the authors employ, we solve this cerainty problem by requiring our statements to be truth-functional. What this means is that we require our labeling words to have direct counterparts in the world of physical existence; the reasoning is anchored in the physical existence of the things referred to. What counts is therefore what can be counted, but what can be counted is only what we can touch and point to. In a nutshell, these are the identity and existence assumptions upon which the language of mathematical and quantitative social science is founded. It should be obvious that these assumptions embody the practice of thingification. It follows that it is only by thinking and speaking about human beings as things rather than as persons that we can make sense out of the Isard-Smith derivations. It should be equally obvious that I am not suggesting that the authors want to treat you and me as things rather than as persons. What I am suggesting is, instead, that by choosing their language the way they have, they have no choice but to treat us accordingly. Their conception of man stems from their use of words.

It may now be wondered why I have taken the trouble of noting that the deep structure of conventional reasoning leads to thingification. I have done it because the authors have practiced it. To be more pointed, I shall argue that they thingify when they perform the conceptual leap which brings them from the realm of formal reasoning to the suggestion that they say something important about what goes on in my head before I take a trip. Implicit in this critique is, of course, the idea that the deep structure of the language within which we perform our inquiries should be the same as the deep structure of the phenomena we are trying to understand. If this is not the case, then some of the aspects we catch in our reasoning net will be distorted, while some others will slip through unnoticed.

This idea of isomorphism between the language we are talking in and the things we are talking about is very important. The reason is that some aspects of what the authors are talking about can be caught in their particular reasoning net. Others cannot. It would seem, for instance, that both the final outcome of our actions and the spatial distribution of the opportunities within which we undertake those actions can be rendered in the language of extensional logic. The case is different, however, when it comes to the human actions that underlie those spatial outcomes. The reason is that human action involves important elements of groping and mental activity. It is exactly this inherent nature of human action that creates problems, for neither groping nor mental objects can be captured in the conventionalist's net. They evade him for two reasons. The first is that whenever we are groping, we violate the concepts of transitivity, separability, and monotonicity. The second is that when we are speaking about mental objects we violate the principle of substitution. To be more specific, groping goes against conventional assumptions of 
identity, while mental phenomena violate the existence assumptions.

What I am suggesting here is that whereas spatial outcomes may well be describable in the language of the Isard-Smith papers, the considerations which lead up to those patterns are not so easily captured. The reason is that the outcomes are surface features anchored in physical objects. The deep structures, on the other hand, are partly in mental phenomena. In the vocabulary of Lévi-Strauss, spatial patterns represent events, while human actions represent structures.

This distinction between structure and event is crucial, for mental and physical phenomena lead existential lives that are as different as God and Devil. In natural languages, we acknowledge this existential difference by referring to physical phenomena by means of so-called oratio recta constructions, whose truth-value is anchored in the objects they refer to. Thus, to take an example, the truth of a transparent sentence like: "It is the case that the spatial pattern is p," depends on the physical truth of $\mathrm{p}$. When we speak about mental phenomena, on the other hand, then we employ so-called oratio obliqua constructions, whose validity is not in the things referred to but rather in the statements themselves. Thus, the validity of the statement: "Isard believes that the pattern $p$ is an optimal pattern," does not depend on whether $p$ actually exists; after all, even Isard's beliefs could be mistaken! Since oratio recta statements deal with physical facts, it follows that they embody the practice of thingification. Rephrased, the distinction is that the truth of transparent statements is in the things which can be counted, while the validity of belief-statements is in the mind of the speaker.

What Isard and Smith can count is the spatial distribution of opportunities and the final outcome of the actors' travel behavior. What they can not count are the beliefs and intentions, the hopes and fears, the agonies and delights which are embedded in those outcomes. Therefore, I conclude that whenever we speak about actors as if they were spatial discounters, then we thingify. Once again, we do not do this because we want to, but because we are forced to by the identity and existence assumptions of the language we are using.

By drawing attention to the deep structure of our reasoning, I wanted to demonstrate how thoroughly imprisoned we are in the language and in the social relations inherent in that language. Our analytical languages are therefore like myths in the sense that our so-called scientific words take on a life of their own; within the fetishism of our invisible college we cannot help but repeat the terms without knowing what they mean and where they come from. My suggestion is that there is much to learn from such people as Wittgenstein and Lévi-Strauss, who have dealt explicitly with the issues of categorization, language, myth, and social relations. Perhaps even the poppy MacLuhan is right on, when he claims that the medium is the message.

But I have an additional reason for discussing the Isard-Smith papers within the framework of language. This is that we may thereby easier come to see the need for another method. That is the method of dialectics. I am saying this because what we do in dialectics is not to criticize the matters of fact we conceive but rather 
the way in which we conceive them. But once I have arrived at the method of dialectics, then I cannot help but be intrigued by the similarities between my characterization of spatial interaction and Marx's characterization of his own society. To illustrate, I shall now take the liberty of selecting a passage from Capital in which I will replace such Marxian key words as man, commodity, value, and money with such regional science terms as regional scientist, geometric pattern, spatial interaction, and human action. The result of this bastardization is as follows:

The [regional scientist's] reflections on the [spatial] forms of social life, and consequently, also, his scientific analysis of those forms, take a course directly opposite to that of their actual historical development. He begins, post festum, with the results of the process ready to hand before him. The characters that stamp [the forms] as [geometric patterns], and whose establishment is a necessary preliminary to [spatial interaction] have already acquired the stability of natural, self-understood forms of social life, before [the regional scientist] seeks to decipher, not their historical character, for in his eyes they are immutable, but their meaning. It is, however, just this ultimate [geometric] form of the world of interaction that actually conceals, instead of disclosing, the social character of [human action], and the social relations between the individual [human beings]. ${ }^{2}$

I have drawn attention to these similarities because throughout his work Marx argued very convincingly, very abstractly and very empirically, that we cannot catch the essence of human action by casting a mechanistic and conventional reasoning net over it. What we capture are only events severed from their deep structures. The lesson is that we will never understand those structures unless we learn how to speak the poetic words of dialectics. The reason is that the batlike words of dialectics are the only ones by which we can capture oblique contexts in which the meaning of what we say changes along with the phenomena we are speaking about. Thus, like the dancer, who follows the rhythm of the music, so the words of the dialectician follow the worlds he refers to.

In conclusion, I will argue that nothing I have said should be construed as negative criticism of the paper and its authors. What they have done is merely to obey the rules of their own game, thereby drawing applause from the crowds of supporters. And that is always an exciting and intoxicating exercise. But to claim that they thereby have helped me understand why two months ago I was in Sweden, why today I am in Chicago, why next week I will be in Toronto, and why next month I will be in New Zealand is another matter. And yet, this seems to be what they claim.

The point is that the authors were misled by their language which requires them to treat you and me as things. But we are not things. Instead we are persons, who have hopes and fears, who cry when we leave and laugh when we meet. To reach

2 The original quote is from Karl Marx, Capital, Vol, J, p. 75f. (New York: International Publishers, 1967). 
an understanding of those aspects of human action, we have no choice but to talk in the fleeting categories of dialectics. To talk about probabilities, as the authors eventually do, is to obfuscate. But to speak about aggregation and testability, which they also do, is important, for aggregation is related to our identity assumptions and testability is embedded in our existence assumptions.

And so it is that we are bound to learn from these papers. As recalled, this is because they carry the seeds of their own destruction; just as Hegel anticipated, the essential nature of the papers turned out to be the reverse of what it wanted to be. But this is nothing to get upset about, for it is merely the way matters are. And since this holds in general, it holds for my own comments as well. 